\title{
Valorization of a Saturated Fatty Acid to Enantioenriched (S)- $\delta$-Decalactone
}

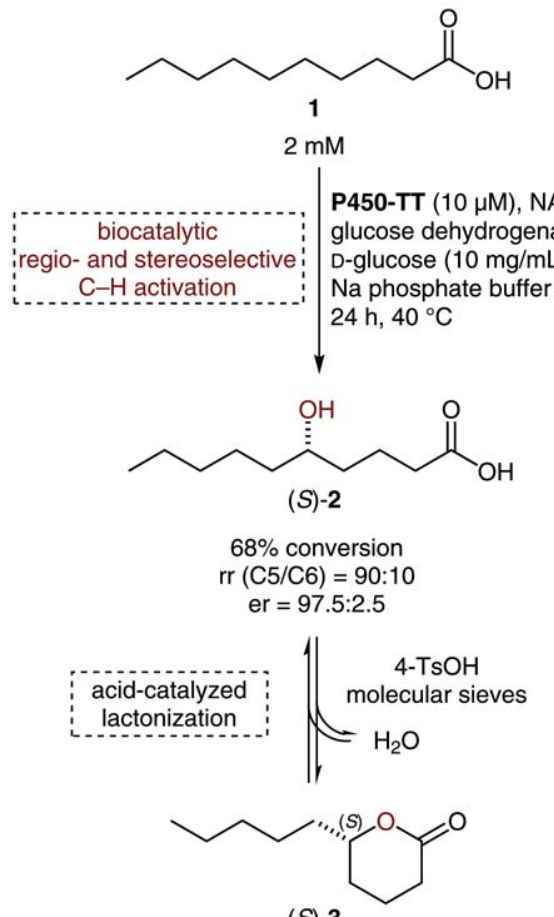

$(S)-3$

er $=97.5: 2.5$

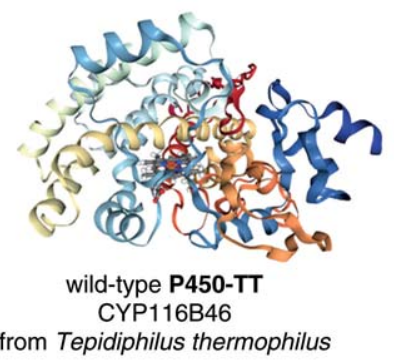

Representation of the docking structure of 1 in P450-TT:

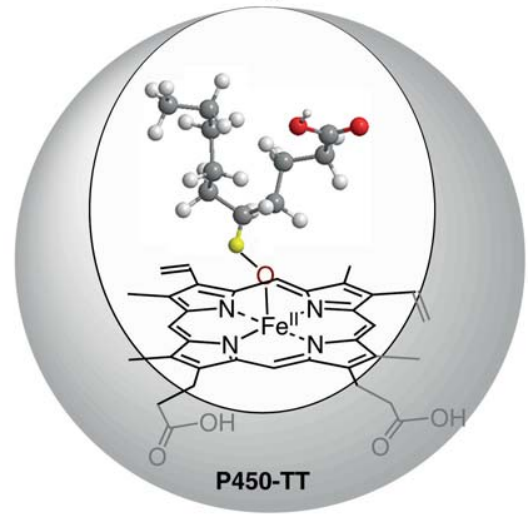

Significance: Hydroxy fatty acids (HFAs) have a wide range of applications as fragrances, food supplements, and pharmaceuticals. The direct, regioand enantioselective $\mathrm{C}-\mathrm{H}$ hydroxylation of nonactivated fatty acids would provide an elegant and efficient approach toward HFAs. Flitsch and co-workers report the first example of a regio- and stereoselective C5 hydroxylation of decanoic acid (1) to give (S)-5-hydroxydecanoic acid (2), catalyzed by a wild-type cytochrome P450 monooxygenase (CYP116B46 from Tepidiphilus thermophilus). Acid-catalyzed cyclization of 2 gave access to the lactonization product (S)- $\delta$-decalactone (3), a high-value fragrance compound.
Comment: Methodologies for the proximal $\alpha$ - and $\beta$-positions or the terminal $\omega-1, \omega-2$ and $\omega$-3-hydroxy acids have been investigated in the past. The mid-chain $\gamma$-and $\delta$-positions have previously been synthesized from functionalized materials. The authors explain the high enantioselectivity of the $\mathrm{C}-\mathrm{H}$ oxyfunctionalization in terms of molecular docking of acid 1 with the active site of P450-TT. Accordingly, substrate 1 folds in a U-shaped conformation and is placed above the heme prosthetic group, permitting hydroxylation in the middle of the chain, giving access to the (S)-enantiomer at C5. It is noteworthy that further engineering of this protein family might enable utilization of a variety of nonactivated substrates. 